\title{
Risk factors for early visual deterioration in temporal arteritis
}

\section{Tobias Loddenkemper, Pankaj Sharma, I Katzan, Gordon T Plant}

J Neurol Neurosurg Psychiatry 2007;78:1255-1259. doi: 10.1136/jnnp.2006.113787

Background: Despite corticosteroid treatment, patients with temporal arteritis may continue to lose vision. However, predictors of progressive visual loss are not known.

Methods: We retrospectively reviewed 341 consecutive patients with suspected temporal arteritis who underwent temporal artery biopsy. 90 patients with biopsy proven temporal arteritis were included in our study.

Results: Twenty-one patients $(23 \%)$ experienced continuous visual symptoms despite steroid therapy and 14 among these suffered persistent visual deterioration. Based on univariate analysis, visual loss on presentation was associated with disc swelling and a history of hypertension. Risk factors for progressive visual loss included older age, elevated $C$ reactive protein and disc swelling.

Conclusion: Although corticosteroid therapy improves the visual prognosis in temporal arteritis, steroids may not stop the progression of visual loss. Our study reliably establishes the risk factors for visual loss in this serious condition. Whether addressing these risk factors early in their presentation can alter the visual outcome remains unknown. Individual risk anticipating treatment regimens and strategies might improve the visual prognosis in temporal arteritis in the future.

eft untreated, temporal arteritis (TA) frequently results in blindness. Treatment requires immediate high dose steroids. -Risk factors for initial and progressive visual loss, despite appropriate treatment, have not been extensively characterised, making selection of patients at risk for progressive visual loss difficult.

We retrospectively reviewed clinical findings in patients with biopsy proven TA in order to assess risk factors for visual loss during the first days of steroid therapy.

\section{METHODS}

A retrospective chart review of 341 patients with suspected TA undergoing temporal artery biopsy during 15 consecutive years at the National Hospital for Neurology and Neurosurgery, London, UK, was performed.

Biopsies were performed on the side of predominant symptoms. Biopsies were deemed positive for TA if the histological specimen demonstrated arteritis, characterised by mononuclear cell arterial wall infiltration and interruption of the internal lamina elastica. Additional evidence included media degeneration or intima thickening. ${ }^{1}$ Visual acuity was expressed as a decimal $(20 / 20=1.00$; finger counting $=0.012$; hand motion $=0.006$; light perception $=0.001$; no light perception $=0){ }^{2}$ In order to compare steroids among patients, the doses in patients receiving a steroid other than hydrocortisone were converted according to the following dosing scheme: $1 \mathrm{mg}$ dexamethasone $=30 \mathrm{mg}$ hydrocortisone; $1 \mathrm{mg}$ methylprednisolone $=5 \mathrm{mg}$ hydrocortisone, $1 \mathrm{mg}$ prednisone $=4 \mathrm{mg}$ hydrocortisone.

SPSS10.0 was used for statistical analysis. A p value of $<0.05$ was considered statistically significant.

\section{RESULTS}

Ninety-three $(27 \%)$ out of 341 biopsy results confirmed TA. Ninety patients (67 females; mean age 74.6 (SD 7.8); range 5993) were included. The remaining three notes were lost.

Onset of symptoms prior to admission ranged from 2 days to 7 years (median 125 days). It took a median of 8 days (0242 days) from referral until admission. Median duration of hospital stay was 11 days (0-53). Ninety-one per cent of biopsies were performed within 5 days of admission; $71 \%$ were biopsied within 5 days after starting steroids. Five patients underwent temporal artery biopsy prior to initiation of steroids. All 90 biopsies were suggestive of TA. Fifty-six (62\%) biopsies showed giant cells.

Eighty-nine patients received corticosteroids. One patient presented 3 weeks after blindness had occurred and was not treated. Thirty-three patients initially received intravenous corticosteroid treatment (hydrocortisone, methylprednisolone or prednisolone). Fifty-six patients were initially treated with prednisone by mouth. Doses were converted to hydrocortisone strength for comparison. The average initial corticosteroid dose consisted of $495 \mathrm{mg}$ of hydrocortisone (range 0-5000). The average time until first dose taper was 21 days. In 31 patients, initial corticosteroid dose was increased in order to control symptoms completely.

Twenty-one $(23 \%)$ patients showed progressive visual symptoms despite corticosteroid therapy. A total of 20 patients suffered visual loss. Thirteen patients suffered persistent loss of acuity or field loss and one additional patient presented with a hemiparesis and diplopia (table 1). Deterioration occurred usually within the first days of treatment. Seven additional patients had transient visual impairment.

Of the seven patients with transient visual impairment despite corticosteroids, four patients had transient visual loss, one patient had an episode of left sided weakness and left visual loss, one patient complained about visual teichopsia and one patient developed disc swelling. All symptoms resolved after an increase in steroid dose.

The presence of disc swelling, history of hypertension and older age were significantly associated with visual loss on presentation. A trend of higher odds for initial visual loss was also seen in patients with polymyalgia, diabetes and jaw claudication (table 2). Patients with visual loss on presentation had a higher systolic blood pressure on presentation (table 3 ).

Risk factors for progressive visual loss included older age, elevated $\mathrm{C}$ reactive protein (CRP) and disc swelling (tables 4 and 5). Fourteen patients with early progressive visual loss despite corticosteroid therapy were, on average, 79.4 years old. Seventy-six patients without progressive visual loss were, on average, 73.7 years old and therefore significantly younger $(\mathrm{p}<0.05$, $\mathrm{t}$ test value 2.2$)$. The CRP of patients with progressive visual loss was, on average, significantly higher $(57.4 ; \mathrm{n}=7$ ) than that of patients without visual loss $(22.1 ; \mathrm{n}=12 ; \mathrm{p}<0.02, \mathrm{t}$ test value -3.0$)$. Other factors significantly $(p<0.05)$ associated with progressive visual loss were disc swelling (odds ratio (OR)

Abbreviations: $A C R$, American College of Rheumatology; CRP, $C$ reactive protein; TA, temporal arteritis 
Table 1 Fourteen patients with loss of visual acuity/fields despite corticosteroid therapy

\begin{tabular}{|c|c|c|c|c|c|c|c|c|c|c|}
\hline \multirow{2}{*}{$\begin{array}{l}\text { Patient } \\
\text { No }\end{array}$} & \multirow[b]{2}{*}{ Sex } & \multirow{2}{*}{$\begin{array}{l}\text { Age } \\
\text { (years) }\end{array}$} & \multirow[b]{2}{*}{ History of present illness } & \multicolumn{2}{|c|}{$\begin{array}{l}\text { Visual acuities } \\
\text { on admission }\end{array}$} & \multirow{2}{*}{$\begin{array}{l}\text { Therapy } \\
\text { prior to } \\
\text { deterioration }\end{array}$} & \multicolumn{2}{|c|}{$\begin{array}{l}\text { Outcome of visual } \\
\text { acuities }\end{array}$} & \multirow{2}{*}{$\begin{array}{l}\text { Time after } \\
\text { therapy } \\
\text { started } \\
\text { (days) }\end{array}$} & \multirow{2}{*}{$\begin{array}{l}2 \text { eyes } \\
\text { involved }\end{array}$} \\
\hline & & & & OD & OS & & OD & OS & & \\
\hline 1 & $\mathrm{~F}$ & 78 & Visual loss OS since 2 days & 0.25 & 0.033 & 60 mg PD po & 0.012 & 0.006 & 5 & Yes \\
\hline 2 & $\mathrm{~F}$ & 79 & Smeary vision OS since 4 days & 0.66 & 0.5 & $\begin{array}{l}200 \mathrm{mg} \mathrm{HC} \text { iv, } \\
40 \mathrm{mg} \mathrm{PD} \mathrm{po}\end{array}$ & 0.66 & 0.006 & 1 & No \\
\hline 3 & $\mathrm{~F}$ & 88 & Visual loss OS same day & 0.333 & 0.006 & $\begin{array}{l}100 \mathrm{mg} \mathrm{HC} \text { iv, } \\
60 \mathrm{mg} \mathrm{PD} \mathrm{po}\end{array}$ & 0.333 & 0 & 1 & No \\
\hline 4 & $\mathrm{~F}$ & 82 & Visual loss OS same day & 0.1 & 0.001 & $\begin{array}{l}200 \mathrm{mg} \mathrm{HC} \text { iv, } \\
60 \mathrm{mg} \text { PD po }\end{array}$ & 0.1 & 0 & 2 & No \\
\hline 5 & $\mathrm{~F}$ & 88 & Visual loss OU same day & 0 & 0.166 & $\begin{array}{l}8 \mathrm{mg} \text { DM iv, } \\
80 \mathrm{mg} \text { PD po }\end{array}$ & 0 & 0.001 & 1 & Yes \\
\hline 6 & $\mathrm{~F}$ & 82 & $\begin{array}{l}\text { Previous PMR } 3 \text { years ago, off } \\
\text { steroids, OD blind } 3 \text { weeks ago, } \\
\text { OS misty since } 3 \text { days }\end{array}$ & 0.001 & 0.001 & $\begin{array}{l}200 \mathrm{mg} \mathrm{HC} \text { iv, } \\
500 \mathrm{mg} \mathrm{MP} \mathrm{iv} \mathrm{x} \\
3 \text { days; } \\
\text { then } 80 \mathrm{mg} P D \text { po }\end{array}$ & 0 & 0 & 6 & Yes \\
\hline \multirow[t]{3}{*}{7} & M & 64 & $\begin{array}{l}\text { Sensory loss right forearm } 3 \text { weeks } \\
\text { ago, double vision since } 2 \text { weeks, } \\
\text { mild right hemiparesis since } 1 \text { day }\end{array}$ & 0.666 & 0.666 & $80 \mathrm{mg}$ PD po & 0.333 & 0.666 & 2 & No \\
\hline & & & & & & $\begin{array}{l}500 \mathrm{mg} \mathrm{MP} \mathrm{iv} \\
120 \mathrm{mg} \text { PD po }\end{array}$ & $\begin{array}{l}0.25 \\
0.166\end{array}$ & $\begin{array}{l}0.666 \\
0.666\end{array}$ & $\begin{array}{l}5 \\
8\end{array}$ & \\
\hline & & & & & & 80 mg PD po & \multicolumn{2}{|c|}{ Hemiparesis deteriorated } & 12 & \\
\hline 8 & $\mathrm{~F}$ & 92 & Loss of vision OD 14 days ago & 0.001 & 0.666 & 60 mg PD po & 0 & 0.666 & 1 & No \\
\hline 9 & $\mathrm{~F}$ & 75 & Visual loss OD since 1 day & 0.5 & 1.0 & 60 mg PD po & 0.001 & 1.0 & 2 & No \\
\hline 10 & M & 87 & Visual loss OD 6 days ago & 0.006 & 1.0 & $\begin{array}{l}500 \mathrm{mg} \mathrm{MP} \text { iv } \mathrm{x} \\
3 \text { days; then } 80 \mathrm{mg} \\
\text { PD po } \\
80 \mathrm{mg} \text { PD po }\end{array}$ & 0.001 & 0.012 & 3 & Yes \\
\hline \multirow[t]{2}{*}{11} & $\mathrm{~F}$ & 80 & $\begin{array}{l}\text { Visual loss OD } 2 \text { weeks ago, } \\
\text { blurred vision OS since } 4 \text { days }\end{array}$ & 0.001 & 0.666 & $\begin{array}{l}500 \mathrm{mg} M P \text { iv } x \\
3 \text { days; then } 80 \mathrm{mg} \\
\text { PD po }\end{array}$ & 0.012 & 0.001 & 1 & Yes \\
\hline & & & & & & $80 \mathrm{mg}$ PD po & 0 & 0 & 10 & Yes \\
\hline 12 & $\mathrm{~F}$ & 75 & $\begin{array}{l}\text { OS blind } 6 \text { days ago, visual loss } \\
\text { OD same day }\end{array}$ & 0.05 & 0 & $\begin{array}{l}1000 \mathrm{mg} \mathrm{MP} \text { iv } \mathrm{x} \\
3 \text { days; then } 80 \mathrm{mg} \\
\text { PD po }\end{array}$ & 0 & 0 & 3 & Yes \\
\hline \multirow[t]{2}{*}{13} & $\mathrm{~F}$ & 59 & $\begin{array}{l}\text { Black spot OS since } 2 \text { days, visual } \\
\text { loss OS same day }\end{array}$ & 1.2 & 0.006 & $80 \mathrm{mg}$ PD po & $\begin{array}{l}0.666 \\
\text { scotoma }\end{array}$ & 0.012 & 2 & Yes \\
\hline & & & & & & $80 \mathrm{mg}$ PD po & $\begin{array}{l}0.666 ; \\
\text { enlarged } \\
\text { scotoma }\end{array}$ & 0.012 & 27 & \\
\hline 14 & $\mathrm{~F}$ & 77 & $\begin{array}{l}\text { PMR since } 3.5 \text { years, visual loss } \\
\text { OS } 6 \text { months ago, visual loss } \\
\text { OD } 2 \text { weeks ago }\end{array}$ & 0.016 & 0.166 & $\begin{array}{l}1000 \mathrm{mg} M P \text { iv } \mathrm{x} \\
3 \text { days; then } 80 \mathrm{mg} \\
\text { PD po }\end{array}$ & 0.012 & 0.1 & 7 & Yes \\
\hline
\end{tabular}

DM, Dexamethasone; HC, hydrocortisone; iv, intravenous; MP, methylprednisolone; OD, right eye; OS, left eye; OU, both eyes; PD, prednisolone; PMR, polymyalgia rheumatica; po, by mouth.

5.3 (95\% CI 1.4 to 20.7)) and administration of steroids intravenously (OR 5.6 (95\% CI 1.6 to 19.9)) (tables 4 and 5). Men had reduced odds of visual loss progression (OR $0.24(95 \%$ CI 0.05 to 1.2$)$ ), approaching statistical significance $(\mathrm{p}=0.06)$ (tables 4 and 5).
Median follow-up duration was 7 months (range 11 days to 16 years). Two year follow-up was available for 28 patients. Four patients without previous visual symptoms had visual loss (unilateral permanent visual loss in two and unilateral temporary visual loss in two). Visual loss occurred, on average, 24 months

Table 2 Risk factors for initial visual loss: dichotomous variable

\begin{tabular}{lcllll}
\hline Dichotomous variable & $\begin{array}{l}\text { Sign present } \\
\text { (\%) }\end{array}$ & $\begin{array}{l}\text { Symptoms/sign } \\
\text { absent } \\
(\%)\end{array}$ & $\chi^{2}$ p value & OR & Cl \\
\hline Disc swelling & 97.6 & 47.9 & 0.000 & 44.56 & $5.66-350.7$ \\
Hypertension & 81.8 & 60.9 & 0.028 & 2.89 & $1.10-7.62$ \\
Polymyalgia & 84.2 & 67.6 & 0.156 & 2.56 & $0.68-9.66$ \\
Diabetes mellitus & 75 & 70.9 & 0.86 & 1.23 & $0.12-12.39$ \\
Jaw claudication & 72.3 & 69.8 & 0.788 & 1.13 & $0.46-2.82$ \\
Diplopia & 70 & 71.4 & 0.901 & 0.93 & $0.31-2.77$ \\
Giant cells on biopsy & 69.6 & 73.5 & 0.693 & 0.83 & $0.32-2.14$ \\
Cholesterol & 100 & 78.6 & 0.605 & 0.79 & $0.6-1.03$ \\
Muscle tenderness & 67.6 & 73.6 & 0.535 & 0.75 & $0.30-1.88$ \\
Male sex & 66.7 & 73.7 & 0.479 & 0.714 & $0.281-1.82$ \\
Stroke & 60 & 71.8 & 0.573 & 0.59 & $0.09-3.76$ \\
Systemic features & 63.6 & 78.3 & 0.126 & 0.49 & $0.19-1.23$ \\
Temple tenderness & 63.6 & 78.3 & 0.126 & 0.49 & $0.19-1.23$ \\
Smoker & 60.5 & 78.8 & 0.058 & 0.41 & $0.16-1.04$ \\
Headache & 67.5 & 92.3 & 0.068 & 0.17 & $0.02-1.41$ \\
\hline
\end{tabular}


Table 3 Risk factors for initial visual loss: continuous variables

\begin{tabular}{lcclll}
\hline Continuous variable & $\begin{array}{l}\text { Mean value in } \\
\text { patients with } \\
\text { visual loss }(\mathbf{n})\end{array}$ & $\begin{array}{l}\text { Mean value in } \\
\text { patients without } \\
\text { visual loss }(\mathbf{n})\end{array}$ & $\begin{array}{l}\text { t test } \\
\text { p value }\end{array}$ & OR & Cl \\
\hline Blood glucose $(\mathrm{mg} / \mathrm{dll})$ & $160(59)$ & $144(22)$ & 0.272 & 1.22 & $1.02-1.46$ \\
Age $(\mathbf{y})$ & $76.6(64)$ & $69.5(26)$ & 0.000 & 1.15 & $1.07-1.24$ \\
Systolic BP $(\mathrm{mm} \mathrm{Hg})$ & $156(64)$ & $145(26)$ & 0.056 & 1.02 & $1.0-1.04$ \\
ESR at referral $(\mathrm{mm})$ & $75(54)$ & $66(18)$ & 0.213 & 1.01 & $0.99-1.04$ \\
Onset (days) & $140(63)$ & $88(26)$ & 0.51 & 1.0 & $0.998-1.00$ \\
Platelets at presentation & $457(50)$ & $448(17)$ & 0.831 & 1.0 & $0.997-1.0$ \\
Corticosteroid dose $(\mathrm{mg} \mathrm{HC)}$ & $672(64)$ & $245(26)$ & 0.004 & 1 & $1.00-1.00$ \\
Time referral to admission (days) & $9(61)$ & $6(24)$ & 0.289 & 1.00 & $0.98-1.02$ \\
ESR at presentation (mm/h) & $70(26)$ & $68(63)$ & 0.779 & 0.996 & $0.98-1.01$ \\
Diastolic BP (mm Hg) & $82(64)$ & $84(26)$ & 0.422 & 0.99 & $0.95-1.03$ \\
Duration of hospital stay & $12(64)$ & $9(26)$ & 0.363 & 1.03 & $0.97-1.09$ \\
\hline BP, blood pressure; ESR, erythrocyte sedimentation rate; HC, hydrocortisone. & & & \\
\hline
\end{tabular}

after initial presentation. All four patients were on a tapering dose of oral prednisone at that time (on average $14 \mathrm{mg}$ daily).

\section{DISCUSSION}

Continuous visual symptoms despite steroid therapy were seen in $23 \%$ of patients, and $16 \%$ suffered visual deterioration during therapy. Risk factors for visual loss on presentation were disc swelling and hypertension. Risk factors for progressive visual loss included older age, elevated CRP and disc swelling.

Fifty-eight patients $(3.1 \%)$ with visual loss after initiation of corticosteroid therapy were found among 1296 patients with TA in the literature (table 6) ranging from $0 \%{ }^{3}$ to $38.9 \%$ in prospective series. ${ }^{4}$ In a meta-analysis of 39 retrospective ${ }^{4-24}$ and prospective studies (table 6), we found a highly significant correlation (Pearson's correlation coefficient 0.604; $\mathrm{p}<0.0001$ ) between the

Table 4 Odds ratios for progressive visual loss: dichotomous symptoms

\begin{tabular}{|c|c|c|c|c|c|}
\hline Symptom (dichotomous) & $\begin{array}{l}\text { Symptom in } \\
\text { patients with } \\
\text { progressive } \\
\text { loss (\%) }\end{array}$ & $\begin{array}{l}\text { Symptom in } \\
\text { patients without } \\
\text { progressive } \\
\text { visual loss (\%) }\end{array}$ & $\chi^{2} \mathrm{p}$ value & OR & $95 \% \mathrm{Cl}$ \\
\hline IV steroids & 30.3 & 7.1 & 0.004 & 5.65 & $1.6-19.91$ \\
\hline Disc swelling & 26.2 & 6.3 & 0.009 & 5.32 & $1.37-20.66$ \\
\hline Stroke & 40 & 14.1 & 0.121 & 4.06 & $0.61-26.86$ \\
\hline Headache & 16.9 & 7.7 & 0.398 & 2.44 & $0.29-20.42$ \\
\hline Scalp tenderness & 20.5 & 10.9 & 0.21 & 2.11 & $0.65-6.81$ \\
\hline Diabetes mellitus & 25 & 15.1 & $0.498^{*}$ & 1.87 & $0.18-19.41$ \\
\hline Systemic symptoms & 18.2 & 13.0 & 0.501 & 1.48 & $0.47-4.68$ \\
\hline Hypertension & 18.2 & 13 & 0.501 & 1.48 & $0.47-4.68$ \\
\hline Muscle tenderness & 16.2 & 15.1 & 0.885 & 1.09 & $0.34-3.45$ \\
\hline Polymyalgia & 15.8 & 15.5 & $0.609^{*}$ & 1.02 & $0.25-4.11$ \\
\hline Cholesterol by history & 0 & 14.3 & $0.867^{*}$ & 0.86 & $0.69-1.06$ \\
\hline Giant cells on biopsy & 14.3 & 17.6 & 0.67 & 0.78 & $0.24-2.47$ \\
\hline Jaw claudication & 12.8 & 18.6 & 0.445 & 0.64 & $0.2-2.02$ \\
\hline Diplopia & 10 & 17.1 & $0.35^{*}$ & 0.537 & $0.11-2.63$ \\
\hline Smoker & 7.9 & 21.2 & 0.086 & 0.32 & $0.08-1.24$ \\
\hline Male sex & 6.1 & 21.1 & 0.059 & 0.242 & $0.051-1.16$ \\
\hline
\end{tabular}

${ }^{*}$ Fisher's exact test.

Table 5 Odds ratios for progressive visual loss: continuous symptoms

\begin{tabular}{|c|c|c|c|c|c|}
\hline Symptom (continuous) & $\begin{array}{l}\text { Mean value in } \\
\text { patients with } \\
\text { progressive } \\
\text { loss (n) }\end{array}$ & $\begin{array}{l}\text { Mean value in } \\
\text { patients without } \\
\text { progressive } \\
\text { visual loss (n) }\end{array}$ & $\begin{array}{l}\text { t test } \\
p \text { value }\end{array}$ & OR & $95 \% \mathrm{Cl}$ \\
\hline CRP on presentation & $57.43(7)$ & $22.17(12)$ & 0.016 & & \\
\hline Age (y) & $79.41(14)$ & $73.7(76)$ & 0.042 & 1.1147 & $1.0208-1.2171$ \\
\hline Glucose (mg/dl) & $155.8(14)$ & $154.5(67)$ & 0.297 & 1.0699 & $0.9009-1.2707$ \\
\hline Diastolic BP $(\mathrm{mm} \mathrm{Hg})$ & $86.79(14)$ & $82.25(76)$ & 0.066 & 1.0371 & $0.9839-1.0932$ \\
\hline Systolic BP (mm Hg) & $159.64(14)$ & $152.15(76)$ & 0.305 & 1.0118 & $0.9894-1.0347$ \\
\hline ESR at referral $(\mathrm{mm} / \mathrm{h})$ & $77.92(13)$ & $71.90(59)$ & 0.466 & 1.01 & $0.99-1.03$ \\
\hline Time referral to admission (days) & $18.29(14)$ & $6.18(71)$ & 0.497 & 1.0093 & $0.9941-1.0247$ \\
\hline ESR at presentation $(\mathrm{mm} / \mathrm{h})$ & $70.79(14)$ & $67.70(76)$ & 0.671 & 1.0044 & $0.9830-1.0263$ \\
\hline Platelets at presentation & $463.36(11)$ & $453.79(56)$ & 0.854 & 1.0004 & $0.9962-1.0047$ \\
\hline Steroid dose (mg HC) & $780(14)$ & $479.21(76)$ & 0.437 & 1.0002 & $0.9998-1.0007$ \\
\hline Onset of symptoms (days) & $109.36(14)$ & $128.35(75)$ & 0.681 & 0.9997 & $0.9975-1.0020$ \\
\hline Length of hospital stay & $21.57(14)$ & $9.13(76)$ & 0.005 & 1.1044 & $1.0403-1.1725$ \\
\hline
\end{tabular}

$\mathrm{BP}$, blood pressure; CRP, C reactive protein; ESR, erythrocyte sedimentation rate; $\mathrm{HC}$, hydrocortisone. *Fisher's exact test. 
Table 6 Visual loss during corticosteroid therapy (prospective studies)

\begin{tabular}{|c|c|c|c|c|c|c|}
\hline Author & Year & $\begin{array}{l}\text { Study design } \\
\text { (cases) }\end{array}$ & $\mathbf{n}$ & Positive biopsy & $\begin{array}{l}\text { Patients with visual } \\
\text { loss (\%) }\end{array}$ & $\begin{array}{l}\text { Patients with ocular } \\
\text { deterioration during } \\
\text { therapy }(\%)\end{array}$ \\
\hline Birkhead $^{33}$ & 1957 & Prospective & 55 & $55(100 \%)$ & $21(38 \%)$ & $2(3.6 \%)$ \\
\hline Palm $^{34}$ & 1958 & Prospective & 31 & $13(21 \%)$ & $31(100 \%)$ & $4(12.9 \%)$ \\
\hline Parsons-Smith ${ }^{35}$ & 1958 & Prospective & 50 (13 treated) & NR & $\begin{array}{l}13 / 55 \text {; all } 13 \text { treated } \\
(24 \%)\end{array}$ & $1(7.7 \%)$ \\
\hline Russell $^{36}$ & 1959 & Retrospective (8) & 35 (21 treated) & $11(31 \%)$ & $\begin{array}{l}16 / 35 \text { with visual } \\
\text { symptoms }(46 \%)\end{array}$ & $6(28.6 \%)$ \\
\hline Mosher $^{4}$ & 1959 & $\begin{array}{l}\text { Prospective (27) } \\
\text { Retrospective }\end{array}$ & 32 (18 treated) & $23(72 \%)$ & $\begin{array}{l}20 \text { with eye symptoms } \\
(62.5 \%)\end{array}$ & $7(38.9 \%)$ \\
\hline Whiffield $^{37}$ & 1963 & Prospective & 72 & NR & $40(55 \%)$ & $1(1.4 \%)$ \\
\hline Cullen ${ }^{38}$ & 1967 & Prospective & 25 & $25(100 \%)$ & $25(100 \%)$ & $1(4 \%)$ \\
\hline Fauchald $^{39}$ & 1972 & Prospective & 94 & $61(65 \%)$ & 5 ocular symptoms (5\%) & $1(1.1 \%)$ \\
\hline Hunder $^{40}$ & 1975 & Prospective & 60 & $60(100 \%)$ & $3(5 \%)$ & $0(0 \%)$ \\
\hline Bengtsson $^{41}$ & 1981 & Prospective & 27 & $17(63 \%)$ & NR & $2(7.4 \%)$ \\
\hline Jones $^{42}$ & 1981 & Prospective & 85 & $22(26 \%)$ & $6 / 22(27 \%)$ permanent & $1(1.2 \%)$ \\
\hline Behn $^{3}$ & 1983 & Prospective & 68 & $25(37 \%)$ & $10(15 \%)$ & $0(0 \%)$ \\
\hline Boesen $^{43}$ & 1987 & Prospective & 21 & 11 (52\%) & $N R$ & $0(0 \%)$ \\
\hline Caselli:i $^{44}$ & 1988 & Prospective & 166 & $166(100 \%)$ & $\begin{array}{l}14 \text { permanent }(8.4 \%) \\
17 \text { transient }\end{array}$ & $1(0.6 \%)$ \\
\hline & & & & & 8 scotoma & \\
\hline $\mathrm{Kyle}^{45}$ & 1989 & Prospective & 35 & NR & NR & $1(2.9 \%)$ \\
\hline Myles $^{46}$ & 1992 & Prospective & $\begin{array}{l}96 \text { TA } \\
210 \text { PMR }\end{array}$ & $\begin{array}{l}48 / 78(61.5 \%) \\
\text { NR }\end{array}$ & NR & $\begin{array}{l}4 / 96(4 \%) \\
3 / 210 \text { PMR (1.4\%) }\end{array}$ \\
\hline Aiello $0^{47}$ & 1993 & Prospective & 245 & 204 (83\%) & $34(14 \%)$ & $5(1.6 \%)$ \\
\hline Duhaut $^{48}$ & 1999 & Prospective & 292 & 207 (71\%) & $31(55 \%)$ & $14(6.8 \%)$ \\
\hline Kupersmith $^{49}$ & 1999 & Prospective & 22 & 19 (86\%) & $7(32 \%)$ & $2(9 \%)$ \\
\hline Chevalet $^{50}$ & 2000 & Prospective & 164 & $128(78 \%)$ & NR & 1 amaurosis $(0.6 \%)$ \\
\hline Kupersmith $^{2}$ & 2001 & Prospective & 20 & $20(100 \%)$ & $4(20 \%)$ & $0(0 \%)$ \\
\hline Liozon $^{29}$ & 2001 & Prospective & 174 & $147(85 \%)$ & $\begin{array}{l}48(28 \%) \text { visual symptoms; } \\
23(13 \%) \text { permanent }\end{array}$ & $4(2.3 \%)$ \\
\hline Danesh-Meyer ${ }^{25}$ & 2005 & Prospective & 34 & $34(100 \%)$ & $34(100 \%)$ & $\begin{array}{l}\text { ( } 27 \% \text { of eyes during the firs } \\
6 \text { days) }\end{array}$ \\
\hline SUM & & & 1838 & $1296(70.5 \%)$ & $362(19.7 \%)$ & $58(3.1 \%)$ \\
\hline
\end{tabular}

percentage of patients with visual loss on presentation and visual loss under corticosteroid therapy (fig 1). Visual loss on presentation may therefore predict visual deterioration under corticosteroid therapy, as also seen in our patients.

Visual deterioration occurs in two peaks. The first peak manifests as progression of the ongoing flare on an unchanged steroid dose, typically during the first 6 days..$^{25}$ The second peak occurs after weeks or months of tapering treatment. Relapses increase with reduction of corticosteroid therapy and were seen in $19 \%$ of patients within 1 year. ${ }^{26}$

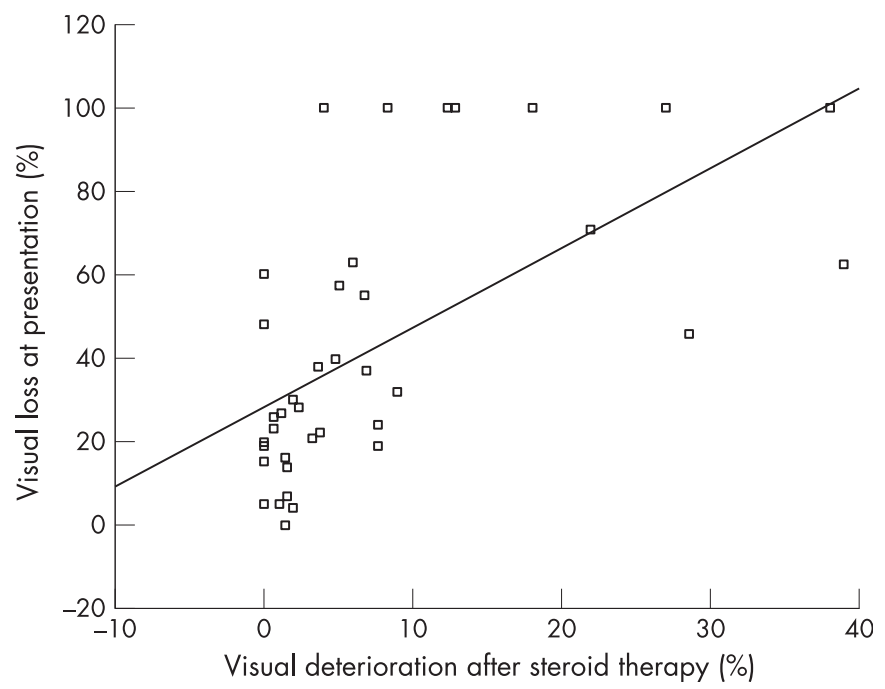

Figure 1 Visual deterioration in relationship to visual loss on presentation, in per cent, based on retrospective and prospective series with complete data from the literature.
Reasons for progression of visual loss despite treatment may include hypoperfusion of the optic disc, treatment delay, inadequate steroid dose, quick taper or hypercoagulability with retinal artery infarction, possibly due to steroid therapy. Continuation of arteritis despite adequate corticosteroid dose may be considered part of the spectrum of TA or may even be a separate disease entity.

Differential diagnoses mimicking TA include systemic lupus erythematodes, Sjögren's syndrome, rheumatoid arthritits, Behcet's disease, antiphospholipid antibody syndrome, polyarteritis nodosa, Churg-Strauss syndrome, Wegener's granulomatosis and other rheumatic conditions presenting with granulomatous vasculitis such as Takyasu arteritis. ${ }^{27}$ Sarcoid, primary angiitis of the central nervous system, non-arteritic AION, neoplastic conditions as well as viral infections (varicella zoster, human parvo virus B19, human herpes virus 6, herpes simplex) and nocardiosis should also be considered.

Risk factors for initial visual loss include transient visual ischaemic symptoms, increased platelet count, jaw claudication and HLA-DRBl phenotype. ${ }^{20} 28$ Constitutional symptoms and elevated liver enzymes are associated with a lower risk of visual loss. ${ }^{28}$ Risk factors for permanent visual loss include amaurosis fugax and cerebrovascular accidents. ${ }^{20}$

Risk factors for progressive visual loss may include occlusive strokes, possibly due to steroid therapy itself. ${ }^{30}$ Late recurrence of visual loss was associated with female sex, older age, worse initial visual acuity, oral (as compared to intravenous) initial steroid treatment and higher erythrocyte sedimentation rate. ${ }^{24}$ HLA DRB 1 alleles were also associated with progressive symptoms. ${ }^{31}$

Intravenous or high dose oral corticosteroids remain the standard of care for patients at risk for visual loss. ${ }^{25} \mathrm{~A}$ retrospective review of 166 patients demonstrated better outcome in patients on low dose aspirin at the time of symptom 
onset. ${ }^{32}$ Aspirin may therefore decrease the rate of visual loss and strokes in patients with TA. Further research is on the way to determine effectiveness. Some authors also use heparin, in particular in patients with progressive visual loss. ${ }^{25}$ Additional steroid sparing agents during the long term treatment period have been tried but no positive randomised placebo controlled prospective trials are available.

Limitations of our study include the retrospective design and referral bias at a tertiary treatment centre. Our literature review attempted to compensate for these variations by comparing our data with previous studies in different settings.

The 1990 American College of Rheumatology (ACR) Criteria for the Classification of Giant Cell (Temporal) Arteritis ${ }^{1}$ were not applied to all 341 patients seen in our clinic because of the retrospective study design. We limited our study population to the gold standard of diagnosis prior to establishment of these criteria, a positive temporal artery biopsy. Nevertheless, all included patients met the 1990 ACR criteria. By making the positive biopsy a prerequisite, we likely applied more sensitive inclusion criteria. Stricter inclusion criteria may also have led to a selection bias towards more active cases. Our data are therefore valid for patients over 50 years old and in whom the biopsy is positive and at least one additional diagnostic ACR criterion is present. A prospective study on progressive visual loss according to ACR standards is needed.

\section{CONCLUSION}

Progression of visual loss despite steroid therapy occurs in a significant minority of patients with TA. In most patients, deterioration occurs within the first 3 days after initiation of steroid therapy. Individual risk anticipating treatment strategies might improve visual prognosis in TA.

\section{Authors' affiliations}

Tobias Loddenkemper, National Hospital for Neurology and

Neurosurgery, London, UK

Gordon T Plant, University Department of Clinical Neurology, Institute of Neurology, London, UK

I Katzan, Department of Neurology, The Cleveland Clinic Foundation, Cleveland, OH, USA

Pankaj Sharma, Department of Clinical Neuroscience, Hammersmith Hospitals and Imperial College, London UK

Competing interests: None.

Correspondence to: Dr Tobias Loddenkemper, Department of Neurology, S51, The Cleveland Clinic Foundation, 9500 Euclid Ave, Cleveland, $\mathrm{OH}$ 44195, USA; loddent@ccf.org

Received 20 December 2006

Revised 7 April 2007

Accepted 12 April 2007

Published Online First 15 May 2007

\section{REFERENCES}

1 Hunder GG, Bloch DA, Michel BA, etal. The American College of Rheumatology 1990 criteria for the classification of giant cell arteritis. Arthritis Rheum 1990;33:1122-8.

2 Kupersmith MJ, Speira R, Langer R, et al. Visual function and quality of life among patients with giant cell (temporal) arteritis. J Neuroophthalmol $2001 ; 21: 266-73$.

3 Behn AR, Perera T, Myles AB. Polymyalgia rheumatica and corticosteroids: how much for how long? Ann Rheum Dis 1983:42:374-8.

4 Mosher HA. Prognosis in temporal arteritis. Arch Ophthalmol 1959;62:641-4.

5 Meadows SP. Temporal arteritis and loss of vision. Trans Ophthal Soc UK 1954;74:13-24.

6 Meadows SP. Temporal or giant cell arteritis. Proc R Soc Med 1966:59:329-33.

7 Healey LA, Wilske KR. Manifestations of giant cell arteritis. Med Clin North Am 1977;60:261-71.

8 Sorensen PS, Lorenzen I. Giant-cell arteritis, temporal arteritis and polymyalgia rheumatica. Acta Med Scand 1977;201:207-13.

9 Huston KA, Hunder GG, Lie JT, et al. Temporal arteritis. A 25-year epidemiologic, clinical and pathologic study. Ann Int Med 1978;88:162-7.

10 Calamia RJ, Hunder GG. Clinical manifestations of giant cell (temporal) arteritis. Clin Rheum Dis 1980;6:403.
11 Fernandez-Herlihy L. Duration of corticosteroid therapy in giant cell arteritis. J Rheumatol 1980;7:361-4.

12 Graham E, Holland A, Avery A, et al. Prognosis in giant-cell arteritis. Br Med J $1981 ; 282: 269-71$.

13 Bengtsson BA. Eye complications in giant cell artertitis. Acta Med Scand 1982;656:S38-43.

14 Delecoevillerie G, Joly P, Cohen de Lara A, et al. Polymyalgia rheumatica and temporal arteritis: a retrospective analysis of prognostic features and different corticosteroid regimens (11 year survey of 210 patients). Ann Rheum Dis 1988:47:733-9.

15 Chmelewski WL, McKnight KM, Agudelo CA, et al. Presenting features and outcomes in patients undergoing temporal artery biopsy. Arch Intern Med 1992;152:1690-5.

16 Liu GT, Glaser JS, Schatz NJ, et al. Visual morbidity in giant cell arteritis. Ophthalmology 1994;101:1779-85.

17 Meli B, Landau K, Gloor BP. The bane of giant cell arteritis from an ophthalmological viewpoint. Schweiz Med Wochenschr 1996;126:1821-8.

18 Font C, Cid MC, Coll-Vincent B, et al. Clinical features in patients with permanent loss due to biopsy-proven giant cell arteritis. Br J Rheumatol 1997;36:251-4.

19 Bahlas S, Ramus-Remus C, Davis C. Clinical outcome of 149 patients with polymyalgia rheumatica and giant cell arteritis. J Rheumatol 1998;25:99-104

20 Gonzalez-Gay MA, Garcia-Porrua C, Llorca J, et al. Visual manifestations of giant cell arteritis. Trends and clinical spectrum in 161 patients. Medicine (Baltimore) 2000;79:283-92.

21 Wenkel $\mathrm{H}$. Bilateral amaurosis in 11 patients with giant cell arteritis confirmed by arterial biopsy. Klin Monatsbl Augenheilkd 2001;218:658-61.

22 Chan CC, Paine $M, O^{\prime}$ Day J. Steroid management in giant cell arteritis. Br J Ophthalmol 2001;85:1061-4.

23 Hayreh SS, Zimmerman B. Management of giant cell arteritis. Our 27-year clinical study: new light on old controversies. Ophthalmologica 2003;217:239-59.

24 Chan CC, Paine M, O'Day J. Predictors of recurrent ischemic optic neuropathy in giant cell arteritis. J Neuroophthalmol 2005;25:14-17.

25 Danesh-Meyer H, Savino PJ, Gamble GG. Poor prognosis of visual outcome after visual loss from giant cell arteritis. Ophthalmology 2005;1 12:1098-103.

26 Hoffman GS, Cid MC, Hellmann DB, et al. A multicenter, randomized, doubleblind, placebo-controlled trial of adjuvant methotrexate treatment for giant cell arteritis. Arthritis Rheum 2002;46:1309-18.

27 Wilke WS. Large vessel vasculitis (giant cell arteritis, Takayasu arteritis). Baillieres Clin Rheumatol 1997;11:285-313.

28 Gonzalez-Gay MA, Blanco R, Rodriguez-Valverde V, et al. Permanent visual loss and cerebrovascular accidents in giant cell arteritis: predictors and response to treatment. Arthritis Rheum 1998;41:1497-504

29 Liozon E, Herrmann F, Ly K, et al. Risk factors for visual loss in giant cell (temporal) arteritis: a prospective study of 174 patients. Am J Med 2001;1 11:211-17

30 Staunton $\mathrm{H}$, Stafford $\mathrm{F}$, Leader $\mathrm{M}$, et al. Deterioration of giant cell arteritis with corticosteroid therapy. Arch Neurol 2000;57:581-4.

31 Rauzy O, Fort M, Nourashemi F, et al. Relation between HLA DRB1 alleles and corticosteroid resistance in giant cell arteritis. Ann Rheum Dis 1998;57:380-2.

32 Nesher G, Berkun Y, Mates M, et al. Low-dose aspirin and prevention of cranial ischemic complications in giant cell arteritis. Arthritis Rheum 2004;50:1332-7.

33 Birkhead NC, Wagener HP, Shick RM. Treatment of temporal arteritis syndrome with adrenal corticosteroids. JAMA 1957;163:821-7.

34 Palm E. The ocular crisis of the temporal arteritis syndrome (Horton). Acta Ophthalmol 1958;36:208-43.

35 Parson-Smith G. Sudden blindness in cranial arteritis. Br J Ophthalmol 1959;43:204-16.

36 Russell RWR. Giant cell arteritis. A review of 35 cases. Q J Med 1959;1 12:471-89.

37 Whiffield AGW, Bateman M, Cooke WT. Temporal arteritis. Br J Ophthalmol 1963;47:555-66.

38 Cullen JF. Ischaemic optic neuropathy. Trans Ophthal Soc UK 1967;87:759-74

39 Fauchald P, Rygrold O, Oystese B. Temporal arteritis and polymyalgia rheumatica. Ann Intern Med 1972;77:845-52.

40 Hunder GG, Sheps SG, Allen LA et al. Daily and alternate-day corticosteroid regimens in treatment of giant cell arteritis. Ann Intern Med 1975;82:613-18.

41 Bengtsson BA, Malmvall BE. The epidemiology of giant cell arteritis including temporal arteritis and polymyalgia rheumatica. Incidences of different clinical presentations and eye complications. Arthritis Rheum 1981;24:899-904.

42 Jones JG, Hazleman BL. Prognosis and management of polymyalgia rheumatica. Ann Rheum Dis 1981;40:1-5.

43 Boesen P, Sorensen SF. Giant cell arteritis, temporal arteritis, and polymyalgia rheumatica in a Danish county. Arthritis Rheum 1987;30:295-9.

44 Caselli RJ, Hunder GG, Whisnant JP. Neurologic disease in biopsy-proven giant cell (temporal) arteritis. Neurology 1988;38:352-9

45 Kyle V, Hazleman BL. Treatment of polymyalgia and giant cell arteritis. I. Steroid regimens for the first two months. Ann Rheum Dis 1989;48:658-61.

46 Myles AB, Perera T, Ridley MG. Treatment of blindness in giant cell arteritis by corticosteroid treatment. Br J Rheumatol 1992;31:103-5.

47 Aiello PD, Trautmann JC, McPhee JT, et al. Visual prognosis in giant cell arteritis. Ophthalmology 1993;100:550-5.

48 Duhaut $\mathrm{P}$, Pinede $\mathrm{L}$, Bornet $\mathrm{H}$, et al. Biopsy proven and biopsy negative temporal arteritis: differences in clinical spectrum at the onset of the disease. Groupe de Recherche sur l'Arterite a Cellules Geantes. Ann Rheum Dis 1999;58:335-41.

49 Kupersmith MJ, Langer $\mathrm{R}$, Mitnick $\mathrm{H}$, et al. Visual performance in giant cell arteritis (temporal arteritis) after 1 year of therapy. Br J Ophthalmol 1999:83:796-801.

50 Chevalet $\mathrm{P}$, Barrier JH, Pottier $\mathrm{P}$, et al. A randomized, multicenter controlled trial using intravenous pulses of methylprednisolone in the initial treatment of simple forms of giant cell arteritis: a one year followup study of 164 patients. J Rheumatol 2000;27:1484-91. 